\title{
Tropomyosin micelles are the major white components in the boiled soup of shellfish
}

Ishida Hideki ( $\sim$ h-ishida@shimane-u.ac.jp)

Shimane University

Takshi Akihiro

Shimane University

Ryo Yasui

Shimane University

Shinji Yasuhira

Iwate Medical University

Ken-ich Matsumoto

Shimane University

Yasuhiro Tanaka

Tottori University

Yasuhiro Matsuo

Shimane University

Hidehisa Shimazu

Shimane University

Takashi Matsuzaki

Shimane University

Shingo Matsumoto

Shimane University

\section{Research Article}

Keywords: Tropomyosin, shellfish, boiled soap, micelles

Posted Date: December 6th, 2021

DOI: https://doi.org/10.21203/rs.3.rs-1139359/v1

License: @ (i) This work is licensed under a Creative Commons Attribution 4.0 International License. Read Full License 


\section{Abstract}

Basket clam soup, a popular Asian dish, is prepared by boiling clams in hot water. The soup is generally cloudy and considered more delicious as cloudiness increases. However, the identity of the whitening ingredients and their relationship with taste remain unclear. In this study, we aimed to identify the components that contribute to the white color of the boiled soup. The white component was precipitated with trichloroacetic acid and reacted positively with ninhydrin, indicating the presence of proteins. The proteins were separated using sodium dodecyl sulfate-polyacrylamide gel electrophoresis, and an intense band was observed at $33 \mathrm{kDa}$. Peptide mass fingerprinting of this band using matrix-assisted laser desorption/ionisation-time-of-flight tandem mass spectrometry revealed the protein to be tropomyosin. Basket clam tropomyosin expressed and purified from Escherichia coli turned the extracted solution white, confirming that tropomyosin contributed to the white color of clam soup.

\section{Introduction}

Basket clams are bivalves that live in freshwater and brackish water. The species that are most widely consumed are Asian clams (Corbicula fluminea and Corbicula leana) and Japanese basket clams (Corbicula japonica). In 2018, the amount of basket clams harvested and consumed was approximately 32,000 tonnes in worldwide, 18,000 tonnes in China, 9,700 tonnes in Japan, and 4,600 tonnes in Taiwan (Food and Agriculture Organization). In the 1920s, the Asian clam was exported from China to the western part of North America as a food item, following which it has now spread to South America and Europe. In particular, the Asian clam (C. fluminea) is one of the "100 worst invading species" in Europe and is recognized as an important alien species that poses a threat to biodiversity and economy ${ }^{1}$. Althogh it is often consumed in Asia, it is rarely eaten in North America, South America, and Europe.

Ben Cao Gamg Mu, a long-used Chinese Compendium of Materia Medica published in 1578, states that "basket clams can prevent alcoholism and cholestasis"2. In Japan, Shokuhin yamato-uta, a collection of "waka" poems about foods published in 1787 states that "basket clams cure jaundice" ${ }^{\text {. }}$ Consumption of basket clams is recommended after drinking excessive amounts of alcohol in Japan. Recent studies have shown that basket clams possess cholesterol-lowering ${ }^{4,5,6}$, cancer-suppressing ${ }^{7,8}$, and hepatoprotective activities ${ }^{9}$.

In Asian countries, basket clams are mainly used as soup ingredients. Basket clam soup (jaechup-guk) is a South Korean delicacy (Figure 1a), and there are many specialty restaurants for basket clams in South Korea. Basket clams are also mainly consumed as soup in China and Taiwan (Figure $1 \mathrm{~b}$, c). Clam extract drinks (Xian jing) are sold in Taiwan, and clear soup and miso soup (shijimi-jiru) prepared using Japanese basket clams ( $C$. japonica) are popular in Japan (Figure 1d).

Typically, basket clams are boiled in hot water before consumption. Boiling turns the clam soup white. Per common belief, the higher the cloudiness, the better the taste. In fact, the cloudiness increases with the number of clams used for cooking. However, the ingredient responsible for the color of the boiled soup remains unidentified. Basket clams are rich in amino acids, such as alanine, glycine, and glutamic acid, and organic acids, such as succinic acid ${ }^{10,11}$, which are believed to contribute to the good taste of the soup. However, high concentrations of these acids do not make the soup cloudy; hence, the relationship between cloudiness and good taste remains unclear.

Therefore, in the current study, we aimed to investigate the identity of the white component in Japanese basket clams as well as other shellfish soups.

\section{Methods}

\section{Materials used}

Japanese basket clams (C. japonica), blood clams (Anadara broughtonii), Asiatic hard clam (Meretrix lusoria), Japanese littleneck (Ruditapes philippinarum), Japanese oysters (Crassostrea nippona), hard clam (Mercenaria mercenaria), Japanese scallop (Mizuhopecten yessoensis), Mediterranean mussel (Mytilus galloprovincialis), banana prawn (Fenneropenaeus merguiensis), and snow crab (Chionoecetes opilio) were obtained from the supermarket.

\section{Formation of cloudy liquid}

One hundred grams of sample was placed in $60 \mathrm{~mL}$ of boiling distilled water for $3 \mathrm{~min}$. The sample was filtered using a filter paper (Qualitative filter papers No. 2, Advantec, Tokyo, Japan) and centrifuged at $8,000 \times g$ for 10 min at $4{ }^{\circ} \mathrm{C}$.

\section{Ultrafiltration}

The sample was ultrafiltered using an Amicon Pro Purification System with a 100-kDa cut-off (Merck Millipore, Tokyo, Japan) by centrifuging at 8,000 × $g$ for $90 \min$ at $4^{\circ} \mathrm{C}$.

\section{Ninhydrin reaction}

Equal volumes of $1 \%$ ninhydrin solution (Fujifilm Wako Pure Chemical, Osaka, Japan) and the sample were mixed and heated in a boiling water bath for 10 min and then allowed to cool to $25{ }^{\circ} \mathrm{C}$. The formation of colour was observed.

\section{Trichloroacetic acid (TCA) precipitation}

Sample and $20 \%$ TCA were mixed in equal amounts, stirred, placed on ice for $10 \mathrm{~min}$, and centrifuged at $10,000 \times \mathrm{g}$ for $10 \mathrm{~min}$ at $4{ }^{\circ} \mathrm{C}$.

\section{Tyndall effect}


A laser pointer (635 nm, 200-LPP029, Sanwa Supply Inc., Okayama, Japan) was used to observe the Tyndall effect.

\section{Electron microscopy}

The boiled soup was stained using a negative staining method. First, one drop of the soup was dropped onto a microgrid covered with a carbon support film hydrophilized via ion sputtering. The microgrid was allowed to stand for $10 \mathrm{~min}$. Next, a $2 \%$ solution of uranyl acetate was added dropwise to the microgrid, and the excess solution was absorbed using a filter paper. The microgrid was dried naturally and observed using a transmission electron microscope (TOPCON Co., Ltd., Tokyo, Japan; EM-002B) at an acceleration voltage of $80 \mathrm{kV}$.

\section{Sodium dodecyl sulfate-polyacrylamide gel electrophoresis (SDS-PAGE) and enzymatic proteolysis}

Protein concentrations were measured using the Bradford method, with bovine serum albumin (Fujifilm Wako Pure Chemical) as the standard. Proteins ( $5 \mu \mathrm{g})$ were separated on a $10 \%(\mathrm{w} / \mathrm{w}$ ) polyacrylamide gel at a constant current of $25 \mathrm{~mA} / \mathrm{gel}$ and stained for 60 min with Coomassie Brilliant Blue R-250 (Fujifilm Wako Pure Chemical) and acetic acid solution. Trypsin ( $50 \mathrm{ng} / \mu \mathrm{L})$ (Promega Madison, WI, USA) was used for digesting all samples. Depending on the volume of the gel pieces, $2-5 \mu \mathrm{L}$ of enzyme solution was added and incubated at $37^{\circ} \mathrm{C}$ for $16 \mathrm{~h}$.

\section{MALDI-TOF-MS/MS}

Trypsin-digested samples were mixed with a-cyano-4-hydroxycinnamic acid and applied to a spot on a mass spectrometry (MS) target plate. MS data were obtained using a 5800 MALDI-TOF/TOF analyzer (ABSciex, Concord, Canada) according to the manufacturer's instructions. A monoisotopic precursor for MS/MS was selected using automatic precursor selection with an interpretation method of the DynamicExit algorithm (AB Sciex). The MS/MS data were analyzed using the ProteinPilot ${ }^{T M}$ software (version 3.0) and the Paragon protein database search algorithm (AB Sciex). Each MS/MS spectrum was searched against the database constructed by AB Sciex.

\section{Absorption spectroscopy}

The absorption spectrum from $200 \mathrm{~nm}$ to $300 \mathrm{~nm}$ was measured using a NanoDrop 1000 spectrophotometer (Thermo Fisher Scientific, Cleveland, OH, USA).

\section{Inductively coupled plasma (ICP)-MS analysis}

The boiled soup was filtered using a 100-kDa ultrafiltration spin column and precipitated using TCA; the pellet was treated with nitric acid and then analyzed using ICP-MS. The S, Na, Mn, Ti, As, Fe, Ni, Cu, Li, Mg, Al, Si, P, K, Ca, Co, Zn, Rb, and Cs contents were determined by ICP-MS (8800 Triple Quadrupole ICP-MS; Agilent, CA, USA), according to the manufacturer's instructions.

\section{Isolation of the tropomyosin gene from $C$. japonica}

Total RNA was extracted from C. japonica using the ISOSPIN Plant RNA kit (Nippon Gene, Tokyo, Japan) and digested with DNase I (NipponGene Co., Ltd., Tokyo, Japan) according to the manufacturer's instructions. A 3- $\mu$ g aliquot of total RNA was used to synthesize single-stranded cDNA using the ReverTra Ace qPCR RT master mix (Toyobo Co., Ltd., Osaka, Japan) according to the manufacturer's instructions. For PCR amplification, the cDNA was denatured at $98^{\circ} \mathrm{C}$ for $3 \mathrm{~min}$ in the first cycle and then for $10 \mathrm{~s}$ in subsequent cycles. Primer annealing and extension were performed at $55^{\circ} \mathrm{C}$ and $72{ }^{\circ} \mathrm{C}$ for $5 \mathrm{~s}$ and $10 \mathrm{~s}$, respectively. Thirty cycles of PCR were performed using the following oligonucleotide primers: $5 \dot{\zeta}$-GCCATCAAGAAGAAGATGCAGGCAATGG-3 $\dot{c}$ and $5 \dot{\zeta}-$ CCAGCCAATTCAGCAAA-3'. The PCR products were fractionated on a $1 \%(\mathrm{v} / \mathrm{w})$ agarose gel, and DNA fragments from agarose gel slices were purified using a DNA fragment purification kit (MagExtractor, Toyobo Co., Ltd.). The purified reverse transcription-polymerase chain reaction (RT-PCR) products were directly sequenced using the BigDye termination kit ver 3.1 (Applied Biosystems, Foster City, CA, USA).

\section{Construction of recombinant Escherichia. coli expressing tropomyosin}

The $C$. japonica tropomyosin gene was amplified using PCR with the appropriate primers (5ל-CATCATCATCATCATATGGATGCCATCAAGAAGAA-3 $\dot{c}$ and $5 \dot{c}$ TATCTAGACTGCAGGTTAATACCCAGCCAATTCAG-3'). The amplicon was cloned into the pColdll expression vector to create pColdll-tropomyosin with a His-tag using the In-fusion ${ }^{\circledR}$ Snap Assembly master mix (Takara Bio Inc., Shiga, Japan) according to the manufacturer's instructions. The construct was sequenced

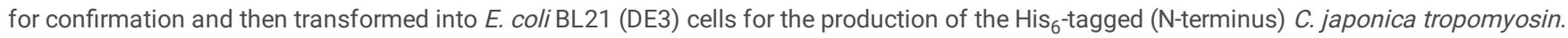

\section{Purification of recombinant proteins}

The transformants were cultured at $37^{\circ} \mathrm{C}$ in Luria Bertani medium containing ampicillin (50 $\left.\mathrm{\mu g} / \mathrm{L}\right)$ (Fujifilm Wako Pure Chemical) until the $\mathrm{OD}_{600}$ was approximately 0.5 . Next, the temperature was changed to $15^{\circ} \mathrm{C}$ to induce cold-shock promoters, and incubation was continued for $24 \mathrm{~h}$. The cells were harvested via centrifugation $\left(6,000 \times \mathrm{g}, 10 \mathrm{~min}, 4^{\circ} \mathrm{C}\right)$ and suspended in $1 \mathrm{~mL}$ of protein extraction reagent (Integral Co., Tokushima, Japan). One microliter of $1,000 \mathrm{U} / \mathrm{mL}$ recombinant DNase I (Takara Bio Inc.) and $0.2 \mathrm{mg} / \mathrm{mL}$ lysozyme (Fujifilm Wako Pure Chemical) were added to each sample and incubated for 15 min at room temperature. Then, the samples were centrifuged $\left(13,000 \times g, 20 \mathrm{~min}, 4^{\circ} \mathrm{C}\right)$. Recombinant proteins were isolated using the His-Spin protein miniprep kit (Zymo Research Co., Irvine, CA, USA), according to the manufacturer's instructions.

\section{Elemental analysis of tropomyosin}

Boiled soup was purified using an Amicon Pro Purification System with a 100-kDa cut-off (Merck Millipore). Samples were collected following TCA precipitation. Subsequently, nitric acid was added to the pellet, and the mixture was incubated at $100{ }^{\circ} \mathrm{C}$ for $12 \mathrm{~h}$. The degraded product was suspended in 0.1 N nitric acid solution and analyzed using ICP-MS (8800 Triple Quadrupole ICP-MS; Agilent). 
Amino acid sequence data of all human proteins were downloaded from Uniprot

(https://ftp.uniprot.org/pub/databases/uniprot/current_release/knowledgebase/reference_proteomes/Eukaryota/UP000005640/UP000005640_9606.fasta.gz Only the canonical entries were used for the following analysis. The amino acid composition was calculated using the Perl script (https://www.nntp.perl.org/group/perl.beginners/2010/12/msg114941.html) with a slight modification (Supplementary Data 1).

\section{Results}

\section{Identification of white ingredients in boiled Japanese basket clam soup}

Initially, Japanese basket clams were boiled and processed to cook the soup (Figure 2a). Ultrafiltration was then performed using a $100 \mathrm{kDa}$ ultrafiltration spin column to identify the substance responsible for the change in color of the boiled water. The obtained flow-through fraction was transparent. The cloudy component was a large molecule and, hence, possibly a protein. Therefore, it was precipitated using TCA or ammonium sulfate, followed by centrifugation. The supernatant obtained was transparent (Figure 2b,c), but the precipitated pellet turned white when dissolved in water (data not shown). The boiled soup turned blue when heated with ninhydrin, an amino acid detection reagent (Figure $2 \mathrm{~d}$ ). Streaks of light were visible when the solution was irradiated with a laser. On the basis of these results, we predicted that the protein formed colloids in this white liquid.

Electron microscopy observations of the negatively stained boiled soup indicated many colloidal particles. The diameter of the smaller particles was $16.0 \mathrm{~nm}$ $\pm 3.2(n=44)$, whereas that of the larger particles was $91.7 n m \pm 19.5(n=45)$ (Figure $2 f, g)$. These particles were smaller than the types of micelles present in milk ${ }^{12}$, which have diameters of approximately $170 \mathrm{~nm}$ and $206 \mathrm{~nm}$ and of which casein is the main component.

To investigate the type of protein present in the boiled soup, the latter was subjected to SDS-PAGE. Ultrafiltration experiments showed that the size of the intended material was larger than $100 \mathrm{kDa}$. However, products larger than $100 \mathrm{kDa}$ were not detected by SDS-PAGE, with a prominent band appearing only at approximately $33 \mathrm{kDa}$ (Figure 3a). The $33 \mathrm{kDa}$ band was recovered from the gel, digested with trypsin, and then subjected to peptide mass fingerprinting using MALDI-TOF-MS/MS. The results revealed the substance to be tropomyosin (accession number P43689) of Biomphalaria glabrata, a freshwater snail (Supplementary Figure 1a). As data on the amino acid sequence of tropomyosin in Japanese basket clams (C. japonica) are lacking, the cDNA of tropomyosin was amplified using RT-PCR. The amino acid sequence of tropomyosin, predicted from the determined nucleotide sequence, is shown in Figure $3 \mathrm{~b}$. The tropomyosin of Japanese basket clams consisted of 284 residues (estimated molecular weight: $32.8 \mathrm{kDa}$ ) (accession number LC564862). Tropomyosin contains only 17 types of amino acids, which exclude tryptophan, histidine, and proline, and include only two residues of cysteine and phenylalanine (Figure 3c). Tropomyosin contains 48 glutamic acid (16.9\%), 32 lysine (11.3\%), 30 leucine (10.5\%), 31 alanine (10.9\%), and 28 aspartic acid (9.8\%) residues (Figure 3c). The proportion of hydrophilic amino acids in tropomyosin was as high as $62 \%$ (Figure $3 \mathrm{~d}$ ). Amino acids with aromatic side chains, such as tryptophan, tyrosine, and phenylalanine, show absorption at $230-300 \mathrm{~nm}$. In particular, absorption by tryptophan is considerable. As the tropomyosin of Japanese basket clams does not contain tryptophan, no or weak absorption was expected, and the absorption of the solution was measured at 200-360 nm. No peak was detected at $280 \mathrm{~nm}$, which is the maximum wavelength of absorption by the protein, whereas a peak was detected at 200-215 nm, which is the maximum absorption wavelength of peptide bonds (Figure 3e).

The MALDI-TOF-MS/MS data were reanalyzed using the expected amino acid sequence of the tropomyosin of Japanese basket clams, and data consistent with eight peptides were obtained (Figure $3 \mathrm{~b}$ colored red square, Supplementary Figure 1b, 1c). Hence, using SDS-PAGE, the detected protein was concluded to be tropomyosin.

The cDNA encoding tropomyosin of Japanese basket clams was cloned into an E. coli protein expression vector (pCOLD2), expressed, and purified. The purified solution became cloudy (Figure $4 \mathrm{~b}$ ) and remained so, even when boiled at $100{ }^{\circ} \mathrm{C}$ for 3 min (Figure $4 \mathrm{~b}$ ). These results showed that tropomyosin caused the cloudiness of the boiled soup.

\section{Characteristics of tropomyosin of Japanese basket clams}

Calcium ions and phosphate are involved in the formation of casein micelles in milk ${ }^{13}$. To investigate whether $\mathrm{Ca}^{2+}$ and phosphate are involved in the formation of micelles in Japanese basket clam soup, the boiled soup was filtered using a $100 \mathrm{kDa}$ ultrafiltration spin column and precipitated using TCA; the pellet was treated with nitric acid and then analyzed using ICP-MS. Calcium ions and phosphate were not detected. The tropomyosin of Japanese basket clams contained three residues of methionine, a sulfur-containing amino acid. The amount of protein can be estimated by measuring the amount of sulfur. Indeed, sulphur and chloride were detected using ICP-MS, although the amount of chloride was only about one-thirteenth that of sulfur (Supplementary Table 1). These results indicate that these elements were not involved in the formation of micelles.

To prevent the formation of hydrophobic bonds in tropomyosin micelles, SDS was added to the boiled soup of Japanese basket clams and heated; however, the extent of cloudiness did not change (Supplementary Figure 2a). Skim milk powder dissolved in water appeared cloudy (Supplementary Figure 2b). To compare clam soup and skim milk solution, SDS was added to the skim milk solution. The cloudiness of the skim milk solution decreased after addition of SDS, and the solution became almost transparent when it was boiled (Supplementary Figure 2b). However, the degree of cloudiness of the clam soup did not change, even when $\beta$-mercaptoethanol was added to disrupt the S-S bond (Supplementary Figure 2c) and urea or guanidine hydrochloride was added to denature proteins (Supplementary Figure 2c3, 2c4). Before performing SDS-PAGE, SDS and $\beta$-mercaptoethanol were added to the boiled soup and boiled; however, the cloudiness persisted and bands above $100 \mathrm{kDa}$ were not detected in SDS-PAGE, except for a band at $33 \mathrm{kDa}$. A white layer was formed when organic solvents such as methanol and acetone were added to the boiled soup (Supplementary Figure 2d), whereas the upper layer became cloudy when chloroform was added (Supplementary Figure 2d). Next, we investigated whether the degree of cloudiness varied with $\mathrm{pH}$. We observed that precipitation 
occurred at $\mathrm{pH} 3-5$. The solution became transparent below pH 3 and the cloudiness almost disappeared at pH 1 (Supplementary Figure 2e). These results indicate that the micelles of tropomyosin are heat-resistant and not denatured by denaturants such as SDS, $\beta$-mercaptoethanol, urea, and guanidine hydrochloride, but are denatured by organic solvents such as methanol, acetone, chloroform, and strong acids.

The same phenomenon was observed in shellfish other than clams, such as blood clams, Asiatic hard clam, Japanese littleneck clam, Japanese oysters, hard clams, Japanese scallop, and Mediterranean mussel (Figure 5a, b). As tropomyosin of crustaceans (shrimp and crab) has been shown to be a heat-resistant protein $^{14}$, similar experiments were performed on banana shrimp and snow crabs. No cloudiness was observed in the snow crab soup (Figure $5 b$ ). Furthermore, cloudiness was not observed when similar experiments were performed on chicken (Gallus domesticus) and fish (Doederleinia berycoides) (data not shown). SDS-PAGE of the boiled soup revealed an intense band at approximately 33-37 kDa for all examined organisms. Similarly, two intense bands were observed in SDS-PAGE of Japanese oyster soup (Figure $5 \mathrm{c} \&$ Supplementary Figure 1d, 1e). The same result was reported for raw oyster (Crassostrea belcheri $)^{15}$. The $37-k D a$ protein has been identified as tropomyosin, but the other band remains to be identified ${ }^{15}$. MALDI-TOF-MS/MS and analysis using ProteinPilot ${ }^{\mathrm{T} M}$ (AB Sciex) revealed that all proteins investigated in this study were tropomyosin.

\section{Discussion}

Basket clam is a popular food item in Asia. Boiled basket clam soup is white and cloudy in appearance; however, the components contributing to its color are not known. In this study, we showed that the whitening of the boiled soup is due to the presence of tropomyosin-forming micelles. Furthermore, the same results were obtained for other shellfish, suggesting that tropomyosin is the causative agent of cloudiness in boiled shellfish soup. To confirm whether tropomyosin confers good taste, amino acids and citric acid, which are thought to be responsible for good taste, were removed by ultrafiltration the obtained supernatant tasted bitter. Therefore, we concluded that tropomyosin does not contribute to the good taste of basket clam soup but turns the soup cloudy.

Proteins are usually denatured, precipitated, or degraded after boiling for 3 min. However, the basket clam tropomyosin forms a micelle in water, even after boiling. The basket clam soup remained white, even after boiling for $24 \mathrm{~h}$. Furthermore, tropomyosin micelles did not precipitate even when stored at $4^{\circ} \mathrm{C}$ for 6 months. Tropomyosin from crabs and shrimp has been reported to be thermostable ${ }^{14}$. In addition, the three-dimensional structure of bird (chicken) tropomyosin showed that the a-helix content is close to $100 \%$ and that it has a coiled coil structure ${ }^{16}$. Proteins that are rich in a-helices have been investigated using statistical tools ${ }^{17}$. Amino acids with high a-helix-forming ability, including glutamate, methionine, alanine, and leucine, are expected to stabilise the ahelix and, thus, the entire protein structure. The proportion of these amino acids in tropomyosin was as high as $39.5 \%$, whereas that of hydrophilic amino acids was $62 \%$ (Figure $3 \mathrm{~d}$ ). Unlike that of glycine, the R-groups of tryptophan and tyrosine are bulky, and destabilize the a-helices. Proline also destabilizes the a-helix owing to its irregular shape. The R-group of proline binds to the nitrogen of the amide group, which causes steric hindrance. Hydrophobic tryptophan, proline, and neutral histidine are not present in the tropomyosin of Japanese basket clams, which contains 284 amino acid residues. When we examined 14,508 human proteins with more than 280 residues, only 12 proteins, including keratin-associated protein and basic salivary proline-rich protein, consisted of 17 or fewer amino acids (constituent amino acids) (Supplementary Table 2). All proteins contained tryptophan, proline, or histidine. These results showed that tropomyosin from Japanese basket clams, which is rich in hydrophilic amino acids and contains many amino acids with high a-helix forming ability, has an extremely rare amino acid composition, which is responsible for its solubility in water.

Tropomyosin from Japanese basket clams is resistant to denaturants. The turbidity of the clam soup persisted, even when boiled with SDS and $\beta$ mercaptoethanol, although a protein band was observed only at $33 \mathrm{kDa}$ in SDS-PAGE. The mechanism via which the micelles decompose is not understood. Although boiled snow crab soup did not become cloudy, protein bands were detected in SDS-PAGE. Therefore, the tropomyosin micelles of crabs, shellfish, and shrimp differed. The tropomyosin of Japanese basket clams is denaturant-resistant, thermostable, and highly hydrophilic, and proteins with such properties are used in various industries. In the future, three-dimensional structure analysis of tropomyosin micelles and amino acid substitution experiments will improve our understanding regarding these proteins, which can be utilized for industrial applications.

Tropomyosin from crustaceans, such as shrimp and crabs, acts as an allergen ${ }^{14,18}$. The amino acid sequences of tropomyosin from Japanese basket clams showed $56.7 \%$ homology with those of banana shrimp and snow crab. Unlike the eight epitopes present in the tropomyosin of shrimp and crab ${ }^{19}$, only one epitope was identified in the tropomyosin of Japanese basket clams (Supplementary Figure 3a red box). The Japanese basket clams is also known as an allergen ${ }^{20,21}$. However, serological and clinical cross-reactivity was not observed between freshwater clam and shrimp ${ }^{21}$. These results indicated that the allergen in Japanese basket clams is not tropomyosin.

Western blotting using a polyclonal antibody against full-length human tropomyosin (GenTex GTX16386) revealed a band in the protein extracted from the myocardium of mouse; however, no band was observed in the Japanese basket clam sample (Supplementary Figure. 3b). This is possibly because the amino acid sequences of human and mice tropomyosin showed $76.4 \%$ homology, whereas the homology between human and Japanese basket clam tropomyosin was only $50.7 \%$. Tropomyosin has a periodic heptad repeat sequence characteristic of coiled coil proteins. The homology between human and Japanese freshwater clam is not high, but the amino acids in the major sites (such as acidic residues, positively charged residues, apolar residues, D-position aspartate137, and alanine residues) were highly conserved (Supplementary Figure $3 \mathrm{~d}$ ). The a and d regions contained more than $70 \%$ hydrophobic amino acids, whereas the b, c, e, and f regions contained 7-32\% hydrophobic amino acid residues. The cloudiness of boiled soup of Japanese freshwater clams was the strongest among the organisms examined. The boiled soup of snow crab contained tropomyosin, but the boiled soup was yellowish instead of white (Figure $5 b)$. The degree of cloudiness varied considerably from species to species. When observing soups other than Japanese freshwater clam soup under an electron microscope, the structure of micelles appeared to be different in each soup (data not shown). The difference in the amino acid sequence of tropomyosin may be related to the darkness of the cloudiness. By conducting amino acid substitution experiments, it may be possible to identify amino acids that are important for cloudiness. Furthermore, by conducting structure analysis using higher-resolution electron microscopy, it will be possible to clarify 
whether the structure of micelles differs between species. Clarifying the thermostability and the mechanism underlying micelle formation would aid in the application of micelles in the development of industrially beneficial thermostable proteins.

The boiled clam soup remained white and cloudy with the addition of SDS and $\beta$-mercaptoethanol even at $100^{\circ} \mathrm{C}$. It has been established that in these conditions, tropomyosin forms micelles sized larger than $100 \mathrm{kDa}$. Interestingly, when separated using SDS-PAGE, a single band was observed corresponding to the $33 \mathrm{kDa}$ molecular weight. However, it is unclear how electrophoresis could separate the highly stable micelles. Later, it was revealed that tropomyosin caused the cloudy appearance in the clam boiled soup. However, the precise reason for the marked thermostability of tropomyosin under boiling conditions remains elusive. Further research is warranted to accurately elucidate the mechanism through which tropomyosin micelles exhibit high thermostability. This knowledge could be extremely useful and beneficial for producing thermostable proteins.

\section{Abbreviations}

ICP-MS, inductively coupled plasma- mass spectrometry; TCA, trichloroacetic acid; SDS-PAGE, sodium dodecyl sulfate-polyacrylamide gel electrophoresis; MALDI-TOF-MS/MS, matrix-assisted laser desorption/ionization-time-of-flight tandem mass spectrometry; RT-PCR, reverse transcription-polymerase chain reaction.

\section{Declarations}

\section{Acknowledgements}

The authors thank Dr. Fei Lin, Sojia Tasi, Jeong Sangchul, and Seo Hee Kyung for providing photos of the clam soup and Ryoko Hara and Maniwa Tomoko for their assistance with MALDI-TOF-MS/MS. Further, the authors thank the Shinjiko Fisheries Cooperative Association for their valuable inputs and the faculty of Life and Environmental Science at Shimane University for financial assistance in publishing this report. We would like to thank Editage (www.editage.com) for English language editing.

\section{Author contributions}

T.A. and H.I. conceived and supervised the project. T.A. and T.M. designed the experiment. T.A. and R.A. conducted the ninhydrin reaction, TCA precipitation, Tyndall effect, and absorption spectroscopy experiments. H.I. performed electron microscopy. T.A., R.Y., and K.M. performed MALDI-TOFMSMS experiments. S.Y. performed the bioinformatics analysis. R.Y., Y.M., and H.S. performed western blot analysis. T.A. and Y.T. performed protein expression analysis using E. coli. S.M. and T.A. performed ICP-MS analysis. T.A. wrote the paper. All authors discussed the results and contributed to the paper.

\section{Competing interests}

The authors declare no competing interests.

\section{Data availability}

Data on protein sequences of tropomyosin from various species are available at https://www.ncbi.nlm.nih.gov/. Data on all human protein sequences can be downloaded from

https://ftp.uniprot.org/pub/databases/uniprot/current_release/knowledgebase/reference_proteomes/Eukaryota/UP000005640/UP000005640_9606.fasta.gz. Source data are provided with this paper.

\section{References}

1. Nentwig, W.; Bacher, S.; Kumschick, S.; Pyšek, P.; Vilà, M. More than "100 worst" alien species in Europe. Biol. Invasions 2017, 20, 1611-1621. DOI: 10.1007/s10530-017-1651-6

2. Li, S. Compendium of Materia Medica: Ben Cao Gang Mu; Beijing: Foreign Languages Press, 1578.

3. Ohtsuga, N. Shyokuhin-yamato-uta, 1787. https://rmda.kulib.kyoto-u.ac.jp/item/rb00003322

4. Laurent, T.; Okuda, Y.; Chijimatsu, T.; Umeki, M.; Kobayashi, S.; Kataoka, Y.; Tatsuguchi, I.; Mochizuki, S.; Oda, H. Freshwater Clam Extract Ameliorates Triglyceride and Cholesterol Metabolism through the Expression of Genes Involved in Hepatic Lipogenesis and Cholesterol Degradation in Rats. Evid. Based Complement. Alternat. Med. 2013, 2013, 830684. DOI: 10.1155/2013/830684.

5. Chijimatsu, T.; Tatsuguchi, I.; Abe, K.; Oda, H.; Mochizuki, S. A freshwater clam (Corbicula fluminea) extract improves cholesterol metabolism in rats fed on a high-cholesterol diet. Biosci. Biotechnol. Biochem. 2008, 72 (10), 2566-2571. DOl: 10.1271/bbb.80257.

6. Takeshi, C.; Iwao, T.; Oda, H.; Mochizuki., S. A basket clam (Corbicula fluminea) extract reduces cholesterol level and hepatic lipids in normal rats and Xenobiotics-induced hypercholesterolemic rats J. Agric. Food Chem. 2009, 57 (8), 3108-3112. DOI: 10.1021/jf803308h

7. Peng, Y. C.; Fwu, L.; Subeq, Y. M.; Tien, C. C.; Lee, R. P. Basket clam extract supplementation improves wound healing by decreasing the tumor necrosis factor alpha level in blood. J. Sci. Food Agric. 2017, 97 (4), 1193-1199. DOI: 10.1002/jsfa.7849

8. Liao, N.; Chen, S.; Ye, X.; Zhong, J.; Wu, N.; Dong, S.; Yang, B.; Liu, D. Antioxidant and anti-tumor activity of a polysaccharide from freshwater clam, Corbicula fluminea. Food Funct. 2013, 4 (4), 539-548. DOI: 10.1039/c2fo30178d. 
9. Huang, K. C.; Wu, W. T.; Yang, F. L.; Chiu, Y. H.; Peng, T. C.; Hsu, B. G.; Liao, K. W.; Lee, R. P. Effects of freshwater clam extract supplementation on time to exhaustion, muscle damage, pro/anti-inflammatory cytokines, and liver injury in rats after exhaustive exercise. Molecules 2013, 18 (4), 3825-3838. DOI: 10.3390/molecules18043825.

10. Enda, H.; Sagane, Y.; Nakazawa, Y.; Sato, H.; Yamazaki, M. Data on free amino acid contents in Japanese basket clams (Corbicula japonica) from Lake Abashiri and Abashirigawa River. Data Brief 2018, 16, 639-643. DOI: 10.1016/j.dib.2017.11.075.

11. Konosu, S.; Shibota, M.; Hashimoto, Y. Concentration of organic acids in shellfish with particular reference to succinic acid. Eiyo To Shokuryo 1967, 20, 186-189. DOI: 10.4327/jsnfs1949.20.186

12. Hristov, P.; Mitkov, I.; Sirakova, D.; Mehandgiiski, I.; Radoslavov, G. Measurement of casein micelle size in raw dairy cattle milk by dynamic light scattering. In: Milk Proteins - From Structure to Biological Properties and Health Aspects, 2016; pp 19-32. DOI: 10.5772/62779

13. Payens, T. A. Association of caseins and their possible relation to structure of the casein micelle. J. Dairy Sci. 1966, 49 (11), 1317-1324. DOI: 10.3168/jds.S0022-0302(66)88088-8.

14. Naqpal, S.; Rajappa, L.; Metcalfe, D. D.; Rao P. V. Isolation and characterization of heat-stable allergens from shrimp (Penaeus indicus). J. Allergy Clin. Immunol. 1989, 83 (1), 26-36. DOI: 10.1016/0091-6749(89)90474-0

15. Yadzir, Z. H.; Misnan, R.; Bakhtiar, F.; Abdullah, N.; Murad, S. Tropomyosin, the major tropical oyster Crassostrea belcheri allergen and effect of cooking on its allergenicity. Allergy Asthma Clin. Immunol. 2015, 11, 30. DOI: 10.1186/s13223-015-0099-4.

16. Rao, J. N., Rivera-Santiago, R., Li, X. E., Lehman, W. \& Dominguez, R. Structural analysis of smooth muscle tropomyosin alpha and beta isoforms. J. Biol. Chem. 287, 3165-3174 (2012). Rao, J. N.; Rivera-Santiago, R.; Li, X. E.; Lehman, W.; Dominguez, R. Structural analysis of smooth muscle tropomyosin a and $\beta$ isoforms. J. Biol. Chem. 2012, 287 (5), 3165-3174. DOI: 10.1074/jbc.M111.307330.

17. Chou, P. Y.; Fasman, G. D. Prediction of the secondary structure of proteins from their amino acid sequence. Adv. Enzymol. Relat. Areas Mol. Biol. 1978, 47, 45-148. DOI: 10.1002/9780470122921.ch2.

18. Liang, Y. L.; Cao, M. J.; Su, W. J.; Zhang, L. J.; Huang, Y. Y.; Liu, G. M. Identification and characterisation of the major allergen of Chinese mitten crab (Eriocheir sinensis). Food Chem. 2008, 111, P998-P1003. DOI: 10.1016/j.foodchem.2008.05.023

19. Shiomi, K. [Current knowledge on molecular features of seafood allergens]. Shokuhin Eiseigaku Zasshi 2010, 51 (4), 139-152. DOI: 10.3358/shokueishi.51.139.

20. Masukane, S.; Haneda, T.; Taketani, T. Non-IgE mediated gastrointestinal food allergy by Shellfish. Jpn. J. Pediatr. Allergy Clin. Immunol. 2018, 32, 772775. DOI: $10.3388 /$ jspaci.32.772

21. Zhang, Y.; Wang, T.; Gao, S.; Morita, E. Novel allergen from the freshwater clam and the related allergy. J. Dermatol. 2012, 39 (7), 672-674. DOI: 10.1111/j.1346-8138.2011.01471.x.

22. Brown, J. H.; Zhou, Z.; Reshetnikova, L.; Robinson, H.; Yammani, R. D.; Tobacman, L. S.; Cohen, C. Structure of the mid-region of tropomyosin: Bending and binding sites for actin. Proc. Natl. Acad. Sci. USA 2005, 102 (52), 18878-18883. DOI: 10.1073/pnas.0509269102.

23. Lehman, W.; Rynkiewicz, M. J.; Moore, J. R. A new twist on tropomyosin binding to actin filaments: perspectives on thin filament function, assembly and biomechanics. J. Muscle Res. Cell Motil. 2020, 41 (1), 23-38. DOI: 10.1007/s10974-019-09501-5.

\section{Figures}


Figure1

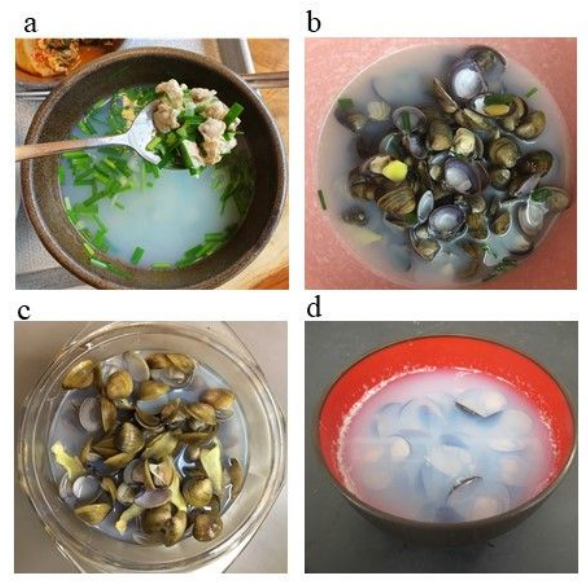

\section{Figure 1}

Soup with basket clam. a, South Korean soup (jaechup-guk). b, Chinese soup (xiăn bèi jiàng tang). c, Taiwanese soup (xiăn tang). d, Japanese soup (shijimijiru). 


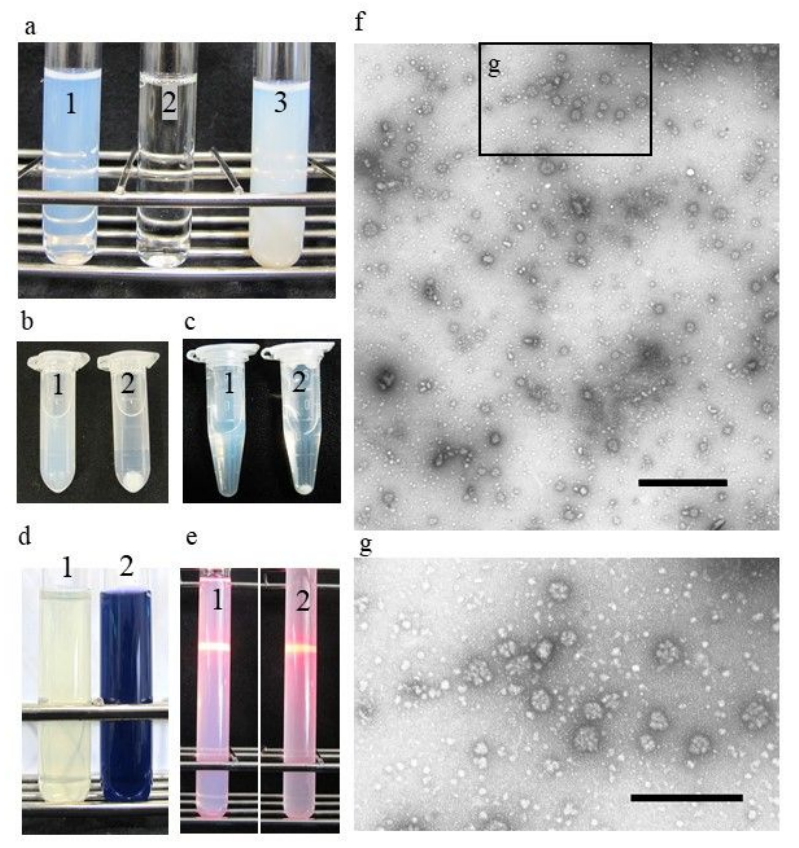

\section{Figure 2}

Analysis of components that whiten boiled clam soup. a, Boiled soup of basket clam (1); Ultrafiltered flow fraction (2); Ultrafiltration supernatant (3); b, TCA was added to a final concentration of $10 \%$ to basket clam boiled soup (1) and centrifuged (2). c, Ammonium sulfate was added to a final concentration of $60 \%$ to basket clam boiled soup (1) and centrifuged (2). d, Ninhydrin was added to a final concentration of $1 \%$ to basket clam boiled soup (1) and heated (2). e, Milk was diluted 1:10 with distilled water and irradiated with a laser beam (1); irradiated basket clam boiled soup (2). f, Negative stain image of boiled soup in transmission electron microscopy. Bar: $1 \mu \mathrm{m}$. g, Enlarged image of particles. Bar: $0.5 \mu \mathrm{m}$ 
Figure 3

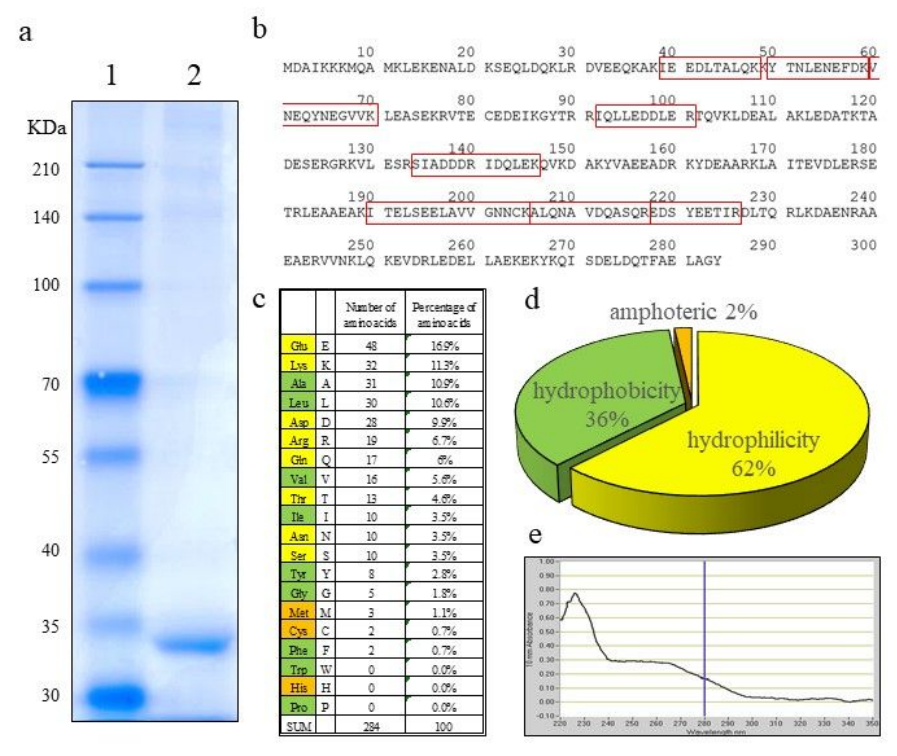

\section{Figure 3}

Identification of proteins by SDS-PAGE, MALDI-TOFMSMS, and amino acid composition of characterized proteins. a, SDS-PAGE of basket clam boiled soup. b, Deduced amino acid sequence of Japanese basket clam (Corbicula japonica) tropomyosin. Amino acids surrounded by red boxes are the parts detected using MALDI-TOF-MS/MS. c, Amino acid composition of Japanese basket clam (Corbicula japonica) tropomyosin. d, Ratio of hydrophilic/hydrophobic/amphoteric amino acids. e, UV absorption of basket clam extract from $220 \mathrm{~nm}$ to $350 \mathrm{~nm}$. 
Figure 4

a

$\begin{array}{llll}\mathrm{KDa} & 1 & 2 & 3\end{array}$

140

100

70

55

40

35

30

20

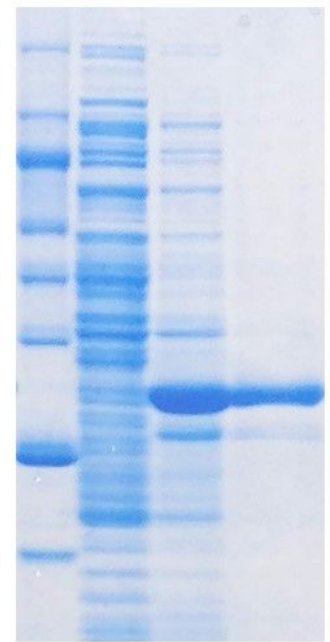

b

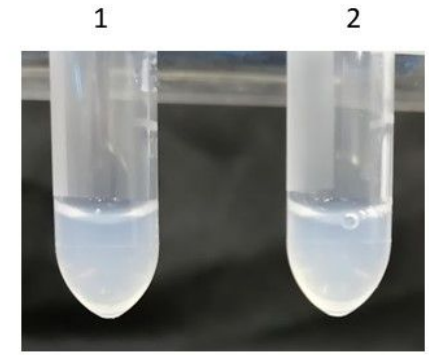

\section{Figure 4}

Expression and purification of tropomyosin in Escherichia coli. a, Tropomyosin from Japanese basket clams (Corbicula japonica) was expressed in Escherichia coli, and the protein was extracted, purified, and subjected to SDS-PAGE. (1) Protein extracted from Escherichia coli under non-inducible conditions

(2) Protein extracted from Escherichia coli under inducible conditions. (3) Purified His-tag protein. b, (1) His-tag purified protein extracted from Escherichia coli.

(2) His-tag purified protein extracted from Escherichia coli boiled for $5 \mathrm{~min}$. 
Figure 5

a

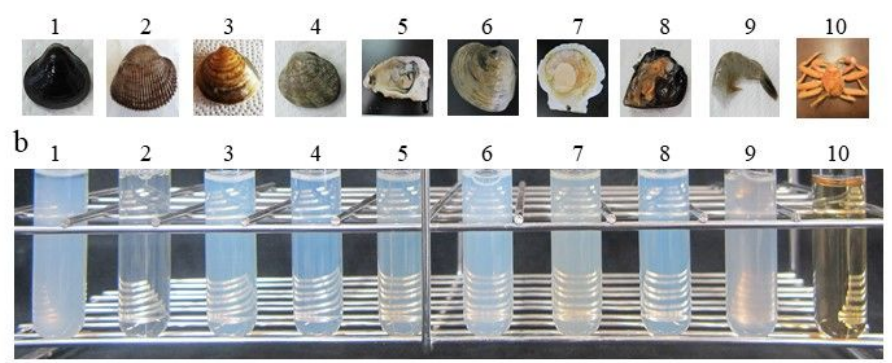

c

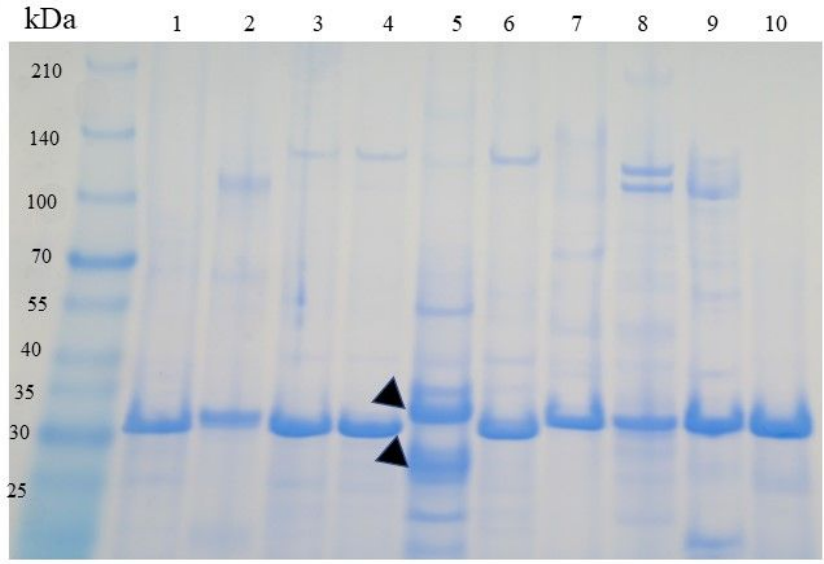

\section{Figure 5}

Boiled soup of various shellfish, shrimp, and crab, and SDS-PAGE analysis of boiled soup. a, Photograph of samples. b, Boiled soups. c, SDS-PAGE of boiled soups. (1) Japanese basket clams (Corbicula japonica). (2) Bloody clam (Anadara broughtonii). (3) Asiatic hard clam (Meretrix lusoria). (4) Japanese littleneck (Venerupis philippinarum). (5) Pacific oyster (Crassostrea gigas). (6) Hard clam (Mercenaria mercenaria). (7) Japanese scallop (Patinopecten yessoensis). (8) Mediterranean mussel (Mytilus galloprovincialis). (9) White leg shrimp (Litopenaeus vannamei). (10) Snow crab (Chionoecetes opilio). The arrows indicate the bands used for MALDI-TOFMS/MS analysis, namely the upper and lower bands.

\section{Supplementary Files}

This is a list of supplementary files associated with this preprint. Click to download.

- SupplementaryFig.1.jpg

- SupplementaryFig.2.jpg

- SupplementaryFig.3.jpg

- SupplementaryTable1.jpg

- SupplementaryTable2.jpg

- Supplementarydata1.xlsx 